\title{
Hepatitis C, A Global Issue: Access to Care and New Therapeutic and Preventive Approaches in Resource-Constrained Areas
}

\author{
Maud Lemoine, MD, $\mathrm{PhD}^{1}$ Mark Thursz, MD, $\mathrm{PhD}^{1}$ \\ ${ }^{1}$ Hepatology \& Gastroenterology Section, Imperial College, London, \\ United Kingdom \\ Address for correspondence Mark Thursz, MD, PhD, Hepatology \& \\ Gastroenterology Section, Imperial College, Norfolk Place, London W2 \\ 1NY, United Kingdom (e-mail: m.thursz@imperial.ac.uk). \\ Semin Liver Dis 2014;34:89-97.
}

Hepatitis C virus (HCV) infection is prevalent in every country where it has been sought making it a global health problem with an estimated prevalence of over 184 million people worldwide. ${ }^{1}$ However, the prevalence rates of infection are highly heterogeneous with a disproportionate burden of infection in countries with limited health care resources. In addition to heterogeneity in the prevalence of infection there is also heterogeneity in the distribution of viral genotypes; in most developed countries genotypes 1 and 3 dominate whereas genotypes 4,5 , and 6 are more common in countries with limited healthcare resources. ${ }^{2}$

Over the past 15 years HCV genotype 1 has been regarded as the most difficult to treat, requiring 48 weeks of pegylated interferon and ribavirin (PegIFN/RBV) and achieving sustained virological response (SVR) rates of only $45 \%$. However, the development of new direct-acting antivirals (DAAs) which are frequently targeted at genotype 1 have made this genotype easier to treat. New IFN-free DAA combinations have also significantly improved SVR rates in HCV genotype 2 and 4 infected patients. However, results are still unsatisfactory in genotype 3 and data in genotypes 5 and 6 are limited.
There are important differences in the dominant routes of transmission between geographical regions with intravenous drug use being the most common route of transmission in Europe, North America, and Australasia and iatrogenic transmission being more important in most resource-limited settings. ${ }^{3}$ Preventive strategies are essential to the control of the HCV epidemic, but the variation in routes of transmission will require a tailored approach to infection control according to the local population needs. The priority in resource-limited countries will be control of transmission in health care facilities whereas the priority in affluent countries will be the interruption of transmission among people who inject drugs (PWID).

Although there is significant variation between countries in the routes of transmission, prevalence and burden of disease there is consistency in resource-limited settings for the poor access to treatment.

\section{Prevalence and Burden of Disease}

Chronic viral hepatitis infections impose a tremendous health burden globally both in terms of mortality and in terms of
$10.1055 / \mathrm{s}-0034-1371082$. ISSN 0272-8087. 
disability adjusted life years lost. The Global Burden of Disease Study separates morbidity and mortality due to cirrhosis from that due to liver cancer although both of these outcomes may be caused by chronic viral hepatitis. ${ }^{4}$ One of the consequences of bundling diseases in this way is that cirrhosis (ranked at number 12) and liver cancer (ranked at number 16) lie outside of the top 10 causes of global mortality. ${ }^{4}$ As a result the burden of disease imposed by viral hepatitis lies outside the international health agenda. When the Millennium Development Goals were set they defined the "major infectious diseases" as human immunodeficiency virus (HIV), tuberculosis, and malaria, which subsequently received the lion's share of health care resources and research funding. ${ }^{5}$ If mortality and morbidity from cirrhosis and liver cancer were combined then viral hepatitis would sit comfortably within the top 10 , above tuberculosis and malaria, and would be more difficult for international health authorities to ignore. ${ }^{6}$

Based on systematic reviews and mathematical modeling research the prevalence of $\mathrm{HCV}$ in 2005 was $2.8 \%$ globally representing over 184 million people. This figure had increased for estimates in 1990, which placed the global prevalence at $2.3 \%$ or 122 million. ${ }^{1}$ Only $\sim 12 \%$ of these infected people live in countries which are sufficiently affluent to be able to provide access to reliable health care resources for treatment of HCV (- Table1). ${ }^{1}$

\section{Asia}

Estimates of the prevalence of chronic hepatitis $\mathrm{C}$ infection in Asian countries are undermined by deficiencies of data or by data generated from unrepresentative populations such as blood donors. At least 14 countries in the Western Pacific and Southeast Asian regions have no reliable data on HCV prevalence, which inevitably means that official prevalence statistics are an underestimate of the true value. Nevertheless, the World Health Organization (WHO) statistics indicate that there are 62 million individuals with chronic HCV in the Western Pacific region and 32 million in the Southeast Asian region, meaning that the majority of infected individuals globally are located in Asia. ${ }^{7-9}$

Prevalence estimates are invariably generated according to country with published values ranging from $0.08 \%$ in Hong Kong to $6.0 \%$ in Vietnam. ${ }^{10}$ Many of the published studies rely on prevalence rates in blood donors rather than community-

Table 1 Regional estimates of HCV prevalence adapted from Mohd Hanafiah et al. ${ }^{1}$

\begin{tabular}{|l|l|l|l|}
\hline Region & $\begin{array}{l}\text { Total population in } \\
\mathbf{2 0 0 5} \text { (millions) }\end{array}$ & $\begin{array}{l}\text { Prevalence } \\
\text { (\%) } \\
\text { (95\% uncertainty } \\
\text { interval) }\end{array}$ & $\begin{array}{l}\text { Estimated number infected } \\
\text { (millions) }\end{array}$ \\
\hline High-income Asia-Pacific & 180 & $1.4(1.2-1.5)$ & 2.4 \\
\hline Central Asia & 77 & $3.8(3.0-4.5)$ & 2.9 \\
\hline East Asia & 1,351 & $3.7(3.1-4.5)$ & 50 \\
\hline South Asia & 1,520 & $3.3(2.6-4.4)$ & 50 \\
\hline Southeast Asia & 57.7 & $2.0(1.7-2.3)$ & 11 \\
\hline Australasia & 24 & $2.7(2.2-.2)$ & 0.6 \\
\hline Caribbean & 42 & $2.1(1.6-2.6)$ & 0.7 \\
\hline Central Europe & 119 & $2.4(2.0-2.8)$ & 2.9 \\
\hline Eastern Europe & 212 & $2.9(2.3-3.5)$ & 6.2 \\
\hline Western Europe & 409 & $2.4(2.2-2.7)$ & 10 \\
\hline Andean Latin America & 50 & $2.0(1.4-2.7)$ & 1 \\
\hline Central Latin America & 216 & $1.6(1.3-1.9)$ & 3.4 \\
\hline Southern Latin America & 58 & $1.6(1.1-2.2)$ & 0.9 \\
\hline Tropical Latin America & 193 & $1.2(1.0-1.4)$ & 2.3 \\
\hline North Africa / Middle East & 420 & $3.6(3.2-4.1)$ & 15 \\
\hline High-Income North America & 337 & $1.3(1.1-1.6)$ & 4.4 \\
\hline Oceana & 8 & $2.6(2.1-3.1)$ & 0.2 \\
\hline Central Sub-Saharan Africa & 87 & $2.1(1.6-3.1)$ & 1.9 \\
\hline East Sub-Saharan Africa & 317 & $2.0(1.6-2.4)$ & 6.1 \\
\hline South Sub-Saharan Africa & 68 & $2.1(1.7-2.5)$ & 1.4 \\
\hline West Sub-Saharan Africa & 303 & $2.8(2.4-3.3)$ & 8.4 \\
\hline World & 6,500 & $2.8(2.6-3.1)$ & 184 \\
\hline
\end{tabular}


based surveys. Although the numbers studied are often very large, blood-donor studies can give an underestimate of population HCV prevalence where the service is based on volunteer donation and prescreening or an overestimate where paid donors are used. A recent systematic review by Nguyen et al summarizes the data available and the populations studied for the Asian region. ${ }^{10}$

Viral genotype distribution is heterogeneous in most parts of Asia. In Japan over $85 \%$ of infections is genotype $1 \mathrm{~b}$. Genotype 1b also dominates in China, Hong Kong, Taiwan, Indonesia, and Vietnam. Genotype 2 is not a frequent genotype in any country, but is found in China, Taiwan, Singapore, and the Philippines. Genotype 3 is the dominant genotype in South Asian countries such as India, Pakistan, and Bangladesh, but also in Thailand. Genotypes 4 and 5 are rarely found in Asia, but genotype 6, which is unique to this region, is found in Cambodia, Laos, Myanmar, Thailand, and Vietnam accounting for more than one third of HCV infections. ${ }^{10,11}$ Treatment response rates for HCV genotype 6 are not well documented. A recent study by Thu Thuy and colleagues found that PegIFN/RBV therapy gave a 60\% SVR after 24 -weeks treatment and $71 \%$ SVR after 48 -weeks treatment. ${ }^{12}$ IFN-based treatment response rates in Asia are generally higher than those seen in Europe and North America due to the higher frequency of favorable IL-28B genotypes in Asian populations.

\section{Middle East and North Africa}

North Africa is recognized as one of the highest prevalence regions for HCV globally with an average $3.6 \%$ of adults infected with HCV. However, within this region Egypt stands out as the country with the highest prevalence, recently estimated at $14.7 \%$ in subjects aged 15 to 59 years with lower prevalence in urban areas (10.3\%) compared with rural areas (18\%). ${ }^{13}$ The rapid emergence of the epidemic in Egypt was originally fueled by the mass treatment of schistosomiasis using the injectable drug tartar emetic during the 1960 s. ${ }^{14}$ As the population who were initially infected have now aged and died, the prevalence rate in Egypt has fallen. Furthermore, there is a profound age cohort effect with much higher prevalence rates in the population over 50 years. Furthermore, the HCV epidemic in Egypt faces significant geographical disparities, the prevalence being highest in the Nile Delta (17.5\%) and lower in Cairo, Alexandria, or Suez (9.5\%) and in Frontier Governates (3.8\%). ${ }^{13}$ However, as the morbidity and mortality from HCV infection depend on the duration of infection, the burden of cirrhosis and hepatocellular carcinoma (HCC) in Egypt is still rising and is expected to produce more than 200,000 deaths from cirrhosis or HCC over the next decade. $^{15}$

\section{Eastern Europe}

The prevalence of HCV in Western European countries is moderately well documented and averages out in adult populations at $2.4 \%$ with a range from $0.1 \%$ in Ireland to $4.5 \%$ in Italy. ${ }^{16}$ In Eastern Europe, documentation of HCV epidemiology is not as well covered, but on the whole the prevalence rates are a little higher at an average of $2.9 \%$.
Lowest estimates of prevalence come from the former Yugoslavian countries at an average of $0.2 \%$ in adult populations. Uzbekistan has the highest prevalence at $13 \%$. In Poland seroprevalence of $\mathrm{HCV}$ is 1.4 to $1.7 \%$ and in Russia $4.5 \%$ of the population are HCV infected. HCV genotypes 1 and 3 dominate across the majority of the European regions. ${ }^{16,17}$ However, several studies have recently reported an increase in the prevalence of HCV genotype 4 infections in Western and Southern European countries such as Greece, Spain, Italy, and France where prevalence rates have been estimated between 10 to $24 \%$ in some areas ${ }^{18-21}$

\section{Latin America}

Very little published data have emerged from Latin America with the majority of studies emerging from the more developed nations: Argentina, Brazil, Mexico, Peru, and Venezuela. Prevalence rates range from 1.5 to $2.9 \%$ in the adult populations. Overall, it is estimated that 6.8 to 8.9 million people in Latin America have chronic HCV infection. HCV genotype 1 is dominant throughout Latin America with approximately equal prevalence of genotypes $1 \mathrm{a}$ and $1 \mathrm{~b} .^{22}$

\section{Sub-Saharan Africa}

Population estimates for the prevalence of chronic HCV infection in sub-Saharan Africa range from $0.1 \%$ in South Africa to $13.4 \%$ in Cameroon. According to WHO statistics, the average prevalence rate in Africa is $5.3 \%$, but this is distorted by the North African countries such as Egypt with a large population and a high prevalence rate. As with other global regions, data on prevalence rates are frequently generated from blood-donor populations, which results in some degree of uncertainty. In addition, there is a general paucity in the number of HCV prevalence studies from the region with no data from Cape Verde, Chad, Namibia, Sierra Leone, Swaziland, and Western Sahara. ${ }^{23,24}$

The distribution of HCV genotypes in Africa is, to some extent, clustered according to geographical location and is also characterized by a considerable HCV subtype diversity. Specific genotypes and subtypes are several centuries old and originated in West Africa (genotype 2$)^{25}$ or Central Africa (genotypes 1 and 4). ${ }^{26}$ In West Africa, genotypes 1 to 3 dominate, whereas in Central Africa genotype 4 is dominant. ${ }^{27}$ Southern Africa, including South Africa, Tanzania, and Zambia, is the only region of the world where genotype 5 is the dominant genotype. There is limited experience in the treatment of patients infected with HCV genotype 5; however, trials suggest that 48 weeks of PegIFN/RBV therapy can achieve SVR rates between 50 and $70 \% .{ }^{28}$

\section{HIV-HCV Coinfection}

Of the 34 million HIV-infected individuals worldwide, 4 to 5 million (5-15\%) are estimated to be coinfected with HCV. The prevalence of HIV-HCV coinfection considerably varies within regions and populations, drug users and men who have sex with men being the two main at-risk groups. Globally, intravenous drug use represents the main mode of HCV transmission in HIV individuals. In Eastern Europe and 
Asia, the HCV infection rate has been reported to be up to 70 to $90 \%$ in HIV-infected patients who are mainly drug users. $^{29,30}$ Almost $70 \%$ of PWID worldwide are infected with $\mathrm{HCV}^{31}$ In contrast, in central Europe, where HIV sexual transmission dominates, the rate of coinfection with HCV is lower estimated between 10 to $15 \%{ }^{32}$ In Sub-Saharan Africa, the number of PWID is limited and HIV transmission is mainly heterosexual associated with a lower rate (under $5 \%$ ) of HIVHCV coinfection. ${ }^{33}$ However, there is a wide variation according the Sub-Saharan African regions and populations (e.g., prisoners), and some studies have reported a high rate of HIV-HCV coinfection over $10 \% .34,35$ In addition, injecting drug use has emerged as a recent concern and is a silent hidden epidemic in several Sub-Saharan African capitals such as Dakar or Dar-Es Salam. ${ }^{36,37}$

HIV infection significantly accelerates the progression of HCV-related liver disease and increases the risk of liver complications. In Western countries, coinfection has become a major cause of morbidity and mortality since the introduction of highly active antiretroviral therapy, and the incidence of cirrhosis and its complications, including HCC, has increased greatly over recent years. ${ }^{38}$ HCC is now responsible for a quarter of liver-related deaths in HIV patients. ${ }^{39}$ Owing to considerable improvements in the management and treatment of HIV/AIDS in resource-limited countries, these regions are likely to face the same alarming mortality rates, with chronic hepatitis becoming a significant clinical problem. Thus, harm reduction services and access to screening and treatment in HIV individuals are critical.

\section{Transmission and Prevention}

$\mathrm{HCV}$ is transmitted parenterally, requiring close contact with infected blood. The major routes for HCV transmission have been well documented ${ }^{3}$ although it is possible that rare causes of transmission have yet to be confirmed. Prior to the identification of HCV blood transfusion was one of the main routes of infection worldwide. Other major routes of transmission include intravenous drug use, medical injections, surgical and dental procedures, and intranasal cocaine. Mother-to-child (vertical) transmission of HCV is well recognized though fortunately not common (4-7\%). Sexual transmission of HCV is extremely rare among heterosexual couples, but is well recognized among men who have sex with men particularly when sexual activity is traumatic and in HIV-infected individuals. ${ }^{3}$

Recognition of HCV as the main cause of posttransfusion hepatitis allowed the development of enzyme-linked immunosorbent assay- (ELISA-) based tests for screening donor blood supplies. Current ELISA tests are highly sensitive and specific and have made a significant impact on the safety of transfusion medicine. HCV antibody screening reduced the risk of $\mathrm{HCV}$ transmission from $7.7 \%$ to 1 in 276,000 donations in the United States by $2002 .{ }^{40}$ However, during the early phase of infection HCV antibodies are not present allowing a window period when the donors HCV status cannot be accurately assessed serologically. This resulted in the development and deployment of nucleic acid testing for HCV RNA in serum. In the United States, this has further reduced the risk of transmitting HCV by transfusion to 1 in 1.9 million donations. There is a stark contrast between transfusion safety in North America and Europe compared with resource-limited settings. Routine testing for transfusion transmissible infections is not conducted at all in 39 countries worldwide. In other countries, the testing of donors for HIV, $\mathrm{HBV}$, and HCV is patchy with priority mostly given to HIV. Where testing is performed there is widespread use of rapid test assays with poor quality control leading to a lack of sensitivity. Nucleic acid testing is extremely uncommon in low- and middle-income countries where the skills and financial resources are not readily available. Even in Egypt, where the size of the HCV epidemic has been acknowledged by the government, only $20 \%$ of the blood supply is subjected to nucleic acid testing. Ensuring the safety of blood and blood products is a vital part of the fight to prevent transmission of HCV and should be given priority by global health authorities.

Transmission of HCV through medical or dental procedures has been documented in virtually all countries. WHO estimates that 2 million new HCV infections each year result from unsafe injections. ${ }^{41}$ Initial reports highlighted the high risk of HCV transmission in hemodialysis patients and subsequently transmission was associated with the use of multidose drug vials, surgery, dental procedures, obstetric care, and gastrointestinal endoscopy. Education of clinicians coupled with the implementation of strict infection control measures has reduced this route of transmission substantially in industrialized countries. Single use hypodermics and drug vials combined with more effective sterilization techniques have helped to ensure that iatrogenic transmission of infection is now a rare event. However, lapses in clinical hygiene procedures are linked to outbreaks of infection reinforcing the need for continued vigilance.

In resource-limited countries iatrogenic transmission has played an important role both in the initiation of HCV epidemics and also in the perpetuation of incident infections. The best example of this was the public health campaign in Egypt to eradicate schistosomiasis using reusable syringes to inject tartar emetic. ${ }^{14}$ However, other mass treatment or vaccination campaigns have also led to a rapid spread of HCV exemplified by a malaria treatment program in Cameroon $^{42}$ and the Democratic Republic of Congo. ${ }^{26}$ Tracing HCV infection to medical or dental procedures is particularly difficult as the majority of new infections are asymptomatic. However, a case-control study in acute hepatitis in Egypt revealed a significantly higher risk of recent attendance at medical facilities, sutures, hypodermic infection or dental work in subjects with acute HCV compared with those with acute hepatitis A virus infections. ${ }^{43}$ Studies in Eastern Europe $^{44}$ and Pakistan ${ }^{45}$ also confirm the importance of poor infection control measures in medical and dental practice as a source of ongoing HCV transmission.

In Western countries, vigilant infection control is now widely accepted as part of normal clinical practice and iatrogenically mediated outbreaks of infection are treated as scandalous. For iatrogenic transmission to be terminated globally, there will need to be a large-scale investment in 
education and awareness campaigns that ensures a similar attitudinal change among medical practitioners which results in better protection for their patients. An additional strategy, advocated by patient groups, is to educate the general public about the dangers associated with reuse of medical instruments, which would empower patients to make their own decisions about infection risk when engaging with medical services.

\section{Access and Barriers to Treatment in Resource-Limited Countries}

The majority of HCV-infected individuals are living in low- or middle-income countries where screening and access to care and treatment are rarely available, and public funding for health care is very limited. Lack of screening, expensive diagnostics, the high cost of the drugs, and the lack of human and technical resources combined with political inertia are the main barriers to access to treatment in resource-poor countries. $^{46}$

\section{Screening and Surveillance}

Chronic viral hepatitis infections are invariably asymptomatic until the late stages of cirrhosis or HCC develop. Identifying cases for treatment therefore relies on awareness of the potential of being infected coupled to diagnostic testing or dependent on screening programs. According to the World Hepatitis Alliance/WHO study conducted in 2010 nearly two-thirds of the world population lives in countries where HCV testing is not accessible. ${ }^{47}$ To date, it is estimated that up to $90 \%$ of HCV infected individuals worldwide are unaware of their HCV-positive status. ${ }^{48,49}$ Identifying and testing at-risk populations for HCV infection in each country represent a critical step in the control of the global burden. Currently, there are no formal recommendations from WHO on population-based screening for HCV. Although population screening is a logical step in public health management of the disease, there have been few pilot studies and no health economic analysis in resource-limited settings. Furthermore, screening may reinforce stigmatization of marginalized populations living in countries where screening is sometimes assimilated to social control. Screening may also create ethical dilemmas where false-positive test results cause unnecessary psychological distress and false-negative results allow the disease to progress in infected patients as well as increasing the risk of further transmission. To minimize these risks, there is an urgent need for affordable pointof-care tests for HCV including rapid assessment of HCV antibody and viral load.

To ascertain accurate estimates on the prevalence of $\mathrm{HCV}$ infection and the burden of disease imposed by the virus it is vital to establish surveillance systems designed to capture data relevant to chronic infection. According to the Global Policy Report on Prevention and control of viral hepatitis ${ }^{50}$ from the WHO, $82.5 \%$ of member-state countries have a hepatitis surveillance system, but a substantially smaller proportion had surveillance systems that are appropriate for HCV. Furthermore, the WHO report is based on responses from only $65 \%$ of member-state countries, and many of those listed as nonresponders are located in high-prevalence regions of the world.

\section{Drug Costs and Availability}

The World Hepatitis Alliance/WHO study reported that $41 \%$ of the world's population lives in countries where no public funding is available for viral hepatitis $\mathrm{B} / \mathrm{C}$ treatment with a particular gap in access to HCV antiviral drugs. ${ }^{47}$ Even in countries where appropriate antiviral drugs are licensed and appear on the essential medicines list, there is limited access to treatment. One of the most important barriers to treatment access is the high cost of anti-HCV drugs. PegIFN/RBV treatment still costs approximately $€ 15,000$ to $€ 25,000$ for a 48 week course in Europe, without taking into account the additional biological and medical costs. DAAs are even more expensive ( $\sim € 20,000-40,000$ per year for telaprevir and boceprevir). Prices of second-generation DAAs are expected to be much higher. As an example, sofosbuvir, which provides excellent results in association or not with IFN has been recently licensed in the United States at US $\$ 70,000$ for a 12 -week course of treatment-US $\$ 1,000 /$ pill. Other IFN-free regimens provide excellent results in HCV monoinfected patients as well as HIV coinfected individuals reaching almost $100 \%$ of SVR and are also expected to be highly expensive. Yet, the simplicity, efficiency, and tolerance of such new drugs are particularly adapted to resource-limited settings and must be urgently considered accessible at lower price in developing countries.

Some low- or middle-income countries have succeeded in negotiating PegIFN/RBV therapy costs with or without the support of international health agencies. A biosimilar of PegIFN (Reiferon Retard, RHEIN-MINAPHARM, Cairo, Egypt) has been developed and commercialized at low prices in Egypt with clinical outcomes similar to proprietary branded PegIFN. The Egyptian hepatitis C program has negotiated a price of $\$ 2000$ for a course of treatment and has been able to provide treatment for more than 200,000 individuals since 2008. ${ }^{51}$ With the support of the Global Fund, the Georgian government has recently started to cover PegIFN/RBV therapy for HIV-HCV coinfected subjects. ${ }^{52}$

Treatment coverage should be improved not only in resource-limited countries, but also in developed countries where less than $20 \%$ of $\mathrm{HCV}$-infected patients receive antiviral therapy. Decreasing the cost of the drugs is urgent for developing countries as well as developed countries, which will not be able to cover all the HCV-treatment-related expenses. This goal is feasible, but will require the support of pharmaceutical companies, international health agencies and donors, governments, and nongovernmental organizations, and the commitment of scientists and physicians.

The HIV/AIDS epidemic has demonstrated that barriers to treatment can be overcome, and lessons from HIV/AIDS must be applied to fighting viral hepatitis. Until the mid-2000s, antiretroviral therapy (ART) cost more than $\$ 10,000$ per patient per year, and treatment was not considered cost effective. ${ }^{53}$ Today, following the strong pressure on policymakers and drug companies by society, this cost has fallen to 
less than $\$ 100$ per person per year. As a result, the number of individuals receiving ART in developing countries has increased considerably from 200,000 in 2000 to 8 million to date. Only $0.1 \%$ of African individuals with HIV received ART in 2000 , compared with $54 \%$ in $2011 .{ }^{54}$

The example set in response to the HIV/AIDS epidemic, allows governments to challenge pharmaceutical companies and empowers international health agencies to achieve widespread access to medications that could now be applied to the HCV epidemic. In 2001, following the Pretoria trial in South Africa against pharmaceutical companies, strong pressure from nongovernmental organizations and civil society led to the Doha Declaration on Trade-Related Aspects of Intellectual Property Rights allowing compulsory licenses for medicines "to protect public health and promote access to medicines for all." ${ }^{55}$ Based on this declaration, each country can now declare a disease as an urgent public health issue in its own territory and produce generic drugs to fight the disease. Using this declaration, numerous countries have improved access to ART, but have also opened access to other treatments. As an example, India has declared liver cancer as an urgent public health issue and now produces sorafenib at a generic price ( $€ 150 /$ month Soranib, Cipla Company, Mumbai, India) much lower than the $€ 4,000$ monthly cost in Western countries.

Moreover, antiviral drugs are often extremely cheap to make. According to Hill et al, the cost of production of sofosbuvir and simeprevir for a 12-week treatment course is estimated, respectively, between \$US68 to \$US136 and \$US130 to $\$$ US326. ${ }^{56}$ Thus, the final selling prices of these drugs are incredibly disproportionate. From a public health perspective, pharmaceutical companies and world trade organizations should have a moral obligation to reduce this unacceptable gap.

There are other alternatives to open access to treatment in poor countries. For example, the creation of a medicines patent pool, which aims at pharmaceutical companies that can register their products in a common health impact fund and sell the drugs at lower prices in resource-poor countries. The fund proposes to assess the impact on population health and provide incentives to firms in exchange. ${ }^{57}$ Following the significant increase in ART coverage associated with a great improvement in the survival of HIV/AIDS patients living in developing countries, HIV/AIDS donors (e.g., The Global Fund to Fight AIDS, Tuberculosis and Malaria [GF] and the Presidents Emergency Plan for Aids Relief [PEPFAR]) should urgently consider access to viral hepatitis treatment for those patients who will now die from end-stage liver disease as observed in developed countries.

\section{Skills and Training}

Although PegIFN/RBV therapy has been demonstrated to be feasible in resource-limited countries with similar SVR rates and side effects than those observed in developed countries, ${ }^{58}$ IFN-based therapy is difficult to implement in developing countries because of numerous constrains: the conditions of IFN storage, the management of side effects that require strong medical support, expensive hematopoiet- ic growth factors or blood transfusions in countries where the blood safety is poor, and finally the necessity to regularly assess HCV viral load and full blood count with limited local laboratory capacities. In addition, in many countries such as in Africa and the Middle East, the benefits to be treated with PegIFN/RBV therapy is low as the majority of individuals are nonresponders to IFN therapy being infected with genotypes 1 or 4 , and being IL28B non-CC carriers.

The shortage of health care workers and facilities and the lack of diagnostic tools create another major barrier to access to treatment in resource-limited countries. The number of liver specialists in low- and middle-income countries is very limited and these countries have often very few diagnostic tools for assessing accurately the severity of the liver disease. Thus, transfer of knowledge, capacities, and diagnostic tools from developed countries to developing countries is urgently needed. The recent innovative tools (e.g., portable ultrasound, transient elastography...), which have dramatically improved the management of viral hepatitis-infected patients in developed countries, should now benefit the resource-poor countries.

\section{Low-Cost Diagnostics}

Accurate measurement of HCV viral load is taken for granted in industrialized countries, but is inaccessible and expensive in resource-limited settings. Viral-load measurement costs between $\$ 75$ and $\$ 200$ per test and is generally provided in tertiary centers where sophisticated laboratory equipment is available to run patent-protected kits sold by European and American diagnostics companies. The availability of robust, reliable, reproducible, and cheap viral load assays is a major barrier to the expansion of HCV treatment programs. A small number of companies (Epistem, www.epistem.co.uk; Alere, www.alere.com; Cepheid, www.cepheid.com) are now developing point-of-care devices that might surmount this barrier in the near future. One potential impact of the new DAA is the ability to simplify viral monitoring during therapy. It is entirely feasible that a single qualitative HCV RNA assay prior to treatment and a second qualitative HCV RNA assay 12 weeks after the end of treatment will suffice for the management of the majority of patients and would impose less demands and expenses on diagnostics services. It is also feasible to perform HCV core antigen detection by enzyme immunoassay at baseline and 12 weeks after the end of therapy because core antigen has been shown to be an accurate surrogate marker of viral replication. ${ }^{59}$ A commercial HCV core antigen assay is available, easy to perform, and cheaper than HCV RNA assays, but with slightly less sensitivity for low viral loads.

\section{Guidelines and Strategies}

Very few developing countries have set up national strategies on HCV infection and when available, these guidelines are often incomplete. ${ }^{47}$ At the international level, WHO has set up only recently a group of international experts to design HCV guidelines; in July 2012, WHO announced a framework for global action for the prevention and control of viral hepatitis. $^{60}$ In 2014 the first WHO HCV guidelines should be 
published. These guidelines may have an impact at the national level and should help governments to support access to screening and treatment for HCV infection in the future.

\section{Political Inertia}

Beyond the preventive and therapeutic measures, successful control of a disease at a global level requires strong political will and societal mobilization. However, since its discovery, HCV infection has not raised the same level of attention that has been deployed to fight HIV/AIDS, malaria, tuberculosis, or even polio in the 1950s. Indeed, HCV infection is a silent disease without obvious visible scars that does not mobilize the political actors. And to date, HCV infection does not receive any significant funding in resource-poor countries. Even in the United States, although HCV causes more deaths and infects four times more individuals than HIV, appropriations for HCV are less than $2 \%$ of resources for research and for medical care related to HIV. ${ }^{61}$

Despite the importance of liver diseases attributable to viral hepatitis infections (-Fig. 1), major contributors to global health developments such as The UK Department for International Development and The Gates Foundation do not prioritize viral hepatitis research or program development. The Global Fund, which provides drugs for the treatment of HIV, tuberculosis, and malaria, has, until recently, been unable to provide medication for viral hepatitis. The Global Fund recently supported the purchase of PegIFN in Georgia for HIV coinfected patients but HCV monoinfected patients

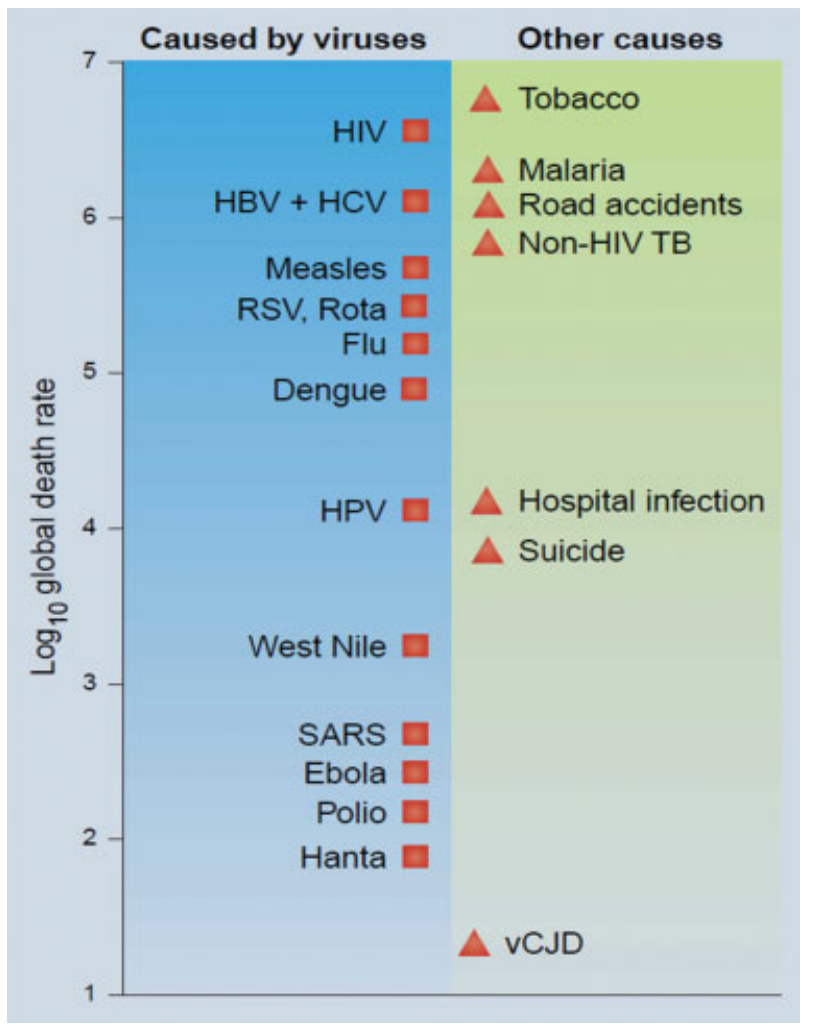

Fig. 1 Putting the mortality from chronic viral hepatitis into context. HBV, hepatitis B virus; HCV, hepatitis C virus; HIV, human immunodeficiency virus; HPV, human papillomavirus; SARS, severe acute respiratory syndrome; vCJD, variant Creutzfeldt-Jakob disease. remain disadvantaged. In the absence of a strong patient lobby, it is unclear what political levers are required to rectify this alarming situation.

\section{Prospects for Treatment in the Future}

All oral DAA with high efficacy and low incidence of side effects promise to change the management of HCV globally. There should no longer be a need for extensive training and skills for the treatment of HCV once we are able to dispense with the side-effect burden imposed by PegIFN/RBV therapy. Furthermore, these drugs will be available for use in patients whom we now consider to be ineligible for treatment, including those who have advanced liver disease or who are intolerant of the IFN-related side effects. The high efficacy rates and the broad genotypic coverage of some of the new drugs will allow treatment regimens to be simplified and obviate the need for sophisticated molecular diagnostics.

From a global health perspective and in the absence of a vaccine, improving access to HCV treatment in low- and middle-income countries has become urgent. By decreasing the pool of transmitters, antiviral therapy also represents an important measure of prevention. To date, with the advent of very highly effective drugs HCV cure has become feasible and global eradication of HCV a new objective. ${ }^{62-64}$ This goal can be achieved, as HCV has no natural nonhuman reservoir, the virus is not integrated in the host genome and is not transmitted by air or food. As a result, transmission can be controlled even in the absence of vaccine with the use of effective antiviral drugs and preventive measures adapted to each country and population.

Three important issues now remain to be addressed for the potential of the new drugs to be realized on a global scale. First, the cost of the new drugs needs to be negotiated to a level that is affordable in resource-limited settings. Second, we need substantial investment in public health research and infrastructure to provide effective means of screening and surveillance for HCV. Third, but most importantly, we need to overcome the political inertia that blocks access to the research funding, health service resources, and financial investment required to tackle the HCV epidemic effectively.

\section{Conclusion}

HCV infection is a leading cause of morbidity and mortality worldwide. The vast majority of HCV-infected individuals are living in resource-limited countries where screening, care, and treatment are rarely accessible. HCV infection has been facing a therapeutic and technologic revolution. For the first time in HCV history, innovative diagnostic tools and highly effective and well-tolerated drugs make an HCV cure and eradication a realistic goal. From public health and health equity perspectives, it is now urgent to overcome the numerous barriers to care and treatment for HCV infection in lowand middle-income countries. Low-cost drugs and diagnostic tools are urgently needed to set up better screening and surveillance, implement treatment programs, and design guidelines adapted to the local settings and populations. To 
be successful, this new HCV era will require a stronger political commitment at international and national levels involving the support of drug companies, patients' groups, and the scientific community.

\section{References}

1 Mohd Hanafiah K, Groeger J, Flaxman AD, Wiersma ST. Global epidemiology of hepatitis $C$ virus infection: new estimates of agespecific antibody to HCV seroprevalence. Hepatology 2013;57(4): 1333-1342

2 Wantuck JM, Ahmed A, Nguyen MH. Review article: the epidemiology and therapy of chronic hepatitis C genotypes 4, 5 and 6 . Aliment Pharmacol Ther 2014;39(2):137-147

3 Thursz M, Fontanet A. HCV transmission in industrialized countries and resource-constrained areas. Nat Rev Gastroenterol Hepatol 2014;11(1):28-35

4 Lozano R, Naghavi M, Foreman K, et al. Global and regional mortality from 235 causes of death for 20 age groups in 1990 and 2010: a systematic analysis for the Global Burden of Disease Study 2010. Lancet 2012;380(9859):2095-2128

5 Lemoine M, Thursz M, Njie R, Dusheiko G. Forgotten, not neglected: viral hepatitis in resource-limited settings, recall for action. Liver Int 2014;34(1):12-15

6 Cooke GS, Lemoine M, Thursz M, et al. Viral hepatitis and the Global Burden of Disease: a need to regroup. J Viral Hepat 2013; 20(9):600-601

7 Sievert W, Altraif I, Razavi HA, et al. A systematic review of hepatitis $C$ virus epidemiology in Asia, Australia and Egypt. Liver Int 2011;31(Suppl 2):61-80

8 Global surveillance and control of hepatitis C. Report of a WHO Consultation organized in collaboration with the Viral Hepatitis Prevention Board, Antwerp, Belgium. J Viral Hepat 1999;6(1):35-47

9 Shepard CW, Finelli L, Alter MJ. Global epidemiology of hepatitis C virus infection. Lancet Infect Dis 2005;5(9):558-567

10 Nguyen LH, Nguyen MH. Systematic review: Asian patients with chronic hepatitis C infection. Aliment Pharmacol Ther 2013; 37(10):921-936

11 Pham DA, Leuangwutiwong P, Jittmittraphap A, et al. High prevalence of Hepatitis C virus genotype 6 in Vietnam. Asian Pac Allergy Immunol 2009;27(2-3):153-160

12 Thu Thuy PT, Bunchorntavakul C, Tan Dat H, Rajender Reddy K. A randomized trial of 48 versus 24 weeks of combination pegylated interferon and ribavirin therapy in genotype 6 chronic hepatitis $C$. J Hepatol 2012;56(5):1012-1018

13 Guerra J, Garenne M, Mohamed MK, Fontanet A. HCV burden of infection in Egypt: results from a nationwide survey. J Viral Hepat 2012;19(8):560-567

14 Frank C, Mohamed MK, Strickland GT, et al. The role of parenteral antischistosomal therapy in the spread of hepatitis $C$ virus in Egypt. Lancet 2000;355(9207):887-891

15 Lehman EM, Wilson ML. Epidemic hepatitis C virus infection in Egypt: estimates of past incidence and future morbidity and mortality. J Viral Hepat 2009;16(9):650-658

16 Hope VD, Eramova I, Capurro D, Donoghoe MC. Prevalence and estimation of hepatitis B and C infections in the WHO European Region: a review of data focusing on the countries outside the European Union and the European Free Trade Association. Epidemiol Infect 2014;142(2):270-286

17 Hatzakis A, Van Damme P, Alcorn K, et al. The state of hepatitis B and $C$ in the Mediterranean and Balkan countries: report from a summit conference. J Viral Hepat 2013;20(Suppl 2):1-20

18 Payan C, Roudot-Thoraval F, Marcellin P, et al. Changing of hepatitis $C$ virus genotype patterns in France at the beginning of the third millenium: The GEMHEP GenoCII Study. J Viral Hepat 2005;12(4): 405-413
19 Ansaldi F, Bruzzone B, Salmaso S, et al. Different seroprevalence and molecular epidemiology patterns of hepatitis $C$ virus infection in Italy. J Med Virol 2005;76(3):327-332

20 Katsoulidou A, Sypsa V, Tassopoulos NC, et al. Molecular epidemiology of hepatitis $C$ virus (HCV) in Greece: temporal trends in HCV genotype-specific incidence and molecular characterization of genotype 4 isolates. J Viral Hepat 2006;13(1):19-27

21 Medrano J, Resino S, Vispo E, et al. Hepatitis C virus (HCV) treatment uptake and changes in the prevalence of HCV genotypes in HIV/HCV-coinfected patients. J Viral Hepat 2011;18(5):325-330

22 Kershenobich D, Razavi HA, Sánchez-Avila JF, et al. Trends and projections of hepatitis $C$ virus epidemiology in Latin America. Liver Int 2011;31(Suppl 2):18-29

23 Karoney MJ, Siika AM. Hepatitis C virus (HCV) infection in Africa: a review. Pan Afr Med J 2013;14:44

24 Madhava V, Burgess C, Drucker E. Epidemiology of chronic hepatitis $C$ virus infection in sub-Saharan Africa. Lancet Infect Dis 2002; 2(5):293-302

25 Markov PV, Pepin J, Frost E, Deslandes S, Labbé AC, Pybus OG. Phylogeography and molecular epidemiology of hepatitis $C$ virus genotype 2 in Africa. J Gen Virol 2009;90(Pt 9):2086-2096

26 Njouom R, Caron M, Besson G, et al. Phylogeography, risk factors and genetic history of hepatitis C virus in Gabon, central Africa. PLoS ONE 2012;7(8):e42002

27 Njouom R, Frost E, Deslandes S, et al. Predominance of hepatitis C virus genotype 4 infection and rapid transmission between 1935 and 1965 in the Central African Republic. J Gen Virol 2009;90(Pt 10):2452-2456

28 Antaki N, Abboud D, Antaki F, Craxi A. HCV genotype 5: an orphan virus. Antivir Ther 2013;18(3):263-269

29 Xia YH, Chen W, Tucker JD, Wang C, Ling L. HIV and hepatitis C virus test uptake at methadone clinics in Southern China: opportunities for expanding detection of bloodborne infections. BMC Public Health 2013;13:899

30 Quan VM, Go VF, Nam V, et al. Risks for HIV, HBV, and HCV infections among male injection drug users in northern Vietnam: a case-control study. AIDS Care 2009;21(1):7-16

31 Nelson PK, Mathers BM, Cowie B, et al. Global epidemiology of hepatitis B and hepatitis C in people who inject drugs: results of systematic reviews. Lancet 2011;378(9791):571-583

32 Rockstroh JK, Mocroft A, Soriano V, et al; EuroSIDA Study Group. Influence of hepatitis $C$ virus infection on HIV-1 disease progression and response to highly active antiretroviral therapy. J Infect Dis 2005;192(6):992-1002

33 Barth RE, Huijgen Q, Taljaard J, Hoepelman AI. Hepatitis B/C and HIV in sub-Saharan Africa: an association between highly prevalent infectious diseases. A systematic review and meta-analysis. Int J Infect Dis 2010;14(12):e1024-e1031

34 Ladep NG, Agaba PA, Agbaji O, et al. Rates and impact of hepatitis on human immunodeficiency virus infection in a large African cohort. World J Gastroenterol 2013;19(10):1602-1610

35 Laurent C, Bourgeois A, Mpoudi-Ngolé E, et al. High rates of active hepatitis B and C co-infections in HIV-1 infected Cameroonian adults initiating antiretroviral therapy. HIV Med 2010;11(1):85-89

36 Bowring AL, Luhmann N, Pont S, et al. An urgent need to scale-up injecting drug harm reduction services in Tanzania: prevalence of blood-borne viruses among drug users in Temeke District, Dar-esSalaam, 2011. Int J Drug Policy 2013;24(1):78-81

37 Raguin G, Lepretre A, Ba I, et al. Drug use and HIV in West Africa: a neglected epidemic. Trop Med Int Health 2011;16(9):1131-1133

38 Joshi D, O'Grady J, Dieterich D, Gazzard B, Agarwal K. Increasing burden of liver disease in patients with HIV infection. Lancet 2011; 377(9772):1198-1209

39 Salmon-Ceron D, Rosenthal E, Lewden C, et al; ANRS EN19 Mortalité Study Group and Mortavic. Emerging role of hepatocellular carcinoma among liver-related causes of deaths in HIV-infected patients: The French national Mortalite 2005 study. J Hepatol 2009;50(4):736-745 
40 Dodd RY, Notari EP IV, Stramer SL. Current prevalence and incidence of infectious disease markers and estimated window-period risk in the American Red Cross blood donor population. Transfusion 2002;42(8):975-979

41 Hauri AM, Armstrong GL, Hutin YJ. The global burden of disease attributable to contaminated injections given in health care settings. Int J STD AIDS 2004;15(1):7-16

42 Pépin J, Lavoie M, Pybus OG, et al. Risk factors for hepatitis C virus transmission in colonial Cameroon. Clin Infect Dis 2010;51(7): 768-776

43 Kandeel AM, Talaat M, Afifi SA, et al. Case control study to identify risk factors for acute hepatitis $C$ virus infection in Egypt. BMC Infect Dis 2012;12:294

44 Chlabicz S, Grzeszczuk A, Prokopowicz D. Medical procedures and the risk of iatrogenic hepatitis $C$ infection: case-controlled study in north-eastern Poland. J Hosp Infect 2004;58(3):204-209

45 Safi SZ, Waheed Y, Sadat J, et al. Molecular study of HCV detection, genotypes and their routes of transmission in North West Frontier Province, Pakistan. Asian Pac J Trop Biomed 2012; 2(7):532-536

46 Lemoine M, Nayagam S, Thursz M. Viral hepatitis in resourcelimited countries and access to antiviral therapies: current and future challenges. Future Virol 2013;8(4):371-380

47 World Hepatitis Alliance. Available at: http://www.who.int/immunization/topics/hepatitis_b_survey_2010/en/. Accessed January 5,2014

48 Hatzakis A, Wait S, Bruix J, et al. The state of hepatitis B and C in Europe: report from the Hepatitis B and C Summit Conference* J Viral Hepat 2011;18(Suppl 1):1-16

49 Thomas DL. Curing hepatitis C with pills: a step toward global control. Lancet 2010;376(9751):1441-1442

50 World Health Organization. Available at: http://www.who.int/csr/ disease/hepatitis/global_report/en/index.html. Accessed January 7,2014

51 Centers for Disease Control and Prevention (CDC). Progress toward prevention and control of hepatitis C virus infection-Egypt, 20012012. MMWR Morb Mortal Wkly Rep 2012;61(29):545-549
52 Eurasian harm reduction network (EHRN) 2013. Available at: http://www.idhdp.com/media/33100/ehrn_hepatitis_c_treatment_access_in_eeca.pdf. Accessed January 5, 2014

53 Marseille E, Hofmann PB, Kahn JG. HIV prevention before HAART in sub-Saharan Africa. Lancet 2002;359(9320):1851-1856

54 UNAIDS. Guidelines for 2014 Global AIDS response progress reporting. Available at: http://www.unaids.org/en/media/unaids/ contentassets/documents/epidemiology/2012/gr2012/ 20121120_UNAIDS_Global_Report_2012_en.pdf. Accessed January 6,2014

55 WTO. The Doha declaration explained. Available at: http://www. wto.org/english/thewto_e/minist_e/min01_e/mindecl_trips_e. pdf. Accessed January 6, 2014

56 Hill A. Minimum costs to provide hepatitis $C$ direct acting antivirals. Available at: http://freepdfhosting.com/d4a7e2bba6.pdf. Accessed January 7, 2014

57 Medicines Patent Pool. Advancing innovation, access, and public health. Available at: http://www.medicinespatentpool.org. Accessed January 7, 2014

58 Ford N, Singh K, Cooke GS, et al. Expanding access to treatment for hepatitis $C$ in resource-limited settings: lessons from HIV/AIDS. Clin Infect Dis 2012;54(10):1465-1472

59 Bouvier-Alias M, Patel K, Dahari H, et al. Clinical utility of total HCV core antigen quantification: a new indirect marker of HCV replication. Hepatology 2002;36(1):211-218

60 WHO. Prevention and control of viral hepatitis infection: framework for global action. Available at: http://www.who.int/csr/ disease/hepatitis/GHP_framework.pdf. Accessed January 6, 2014

61 Edlin BR. Perspective: test and treat this silent killer. Nature 2011; 474(7350):S18-S19

62 Hagan LM, Wolpe PR, Schinazi RF. Treatment as prevention and cure towards global eradication of hepatitis $C$ virus. Trends Microbiol 2013;21(12):625-633

63 Hagan LM, Schinazi RF. Best strategies for global HCV eradication. Liver Int 2013;33(Suppl 1):68-79

64 Thomas DL. Global control of hepatitis C: where challenge meets opportunity. Nat Med 2013;19(7):850-858 